\title{
Inflection, canards and folded singularities in excitable systems - Application to a 3D FitzHugh-Nagumo model
}

\author{
J. Uria Albizuri • M. Desroches • \\ M. Krupa • S. Rodrigues
}

Received: date / Accepted: date

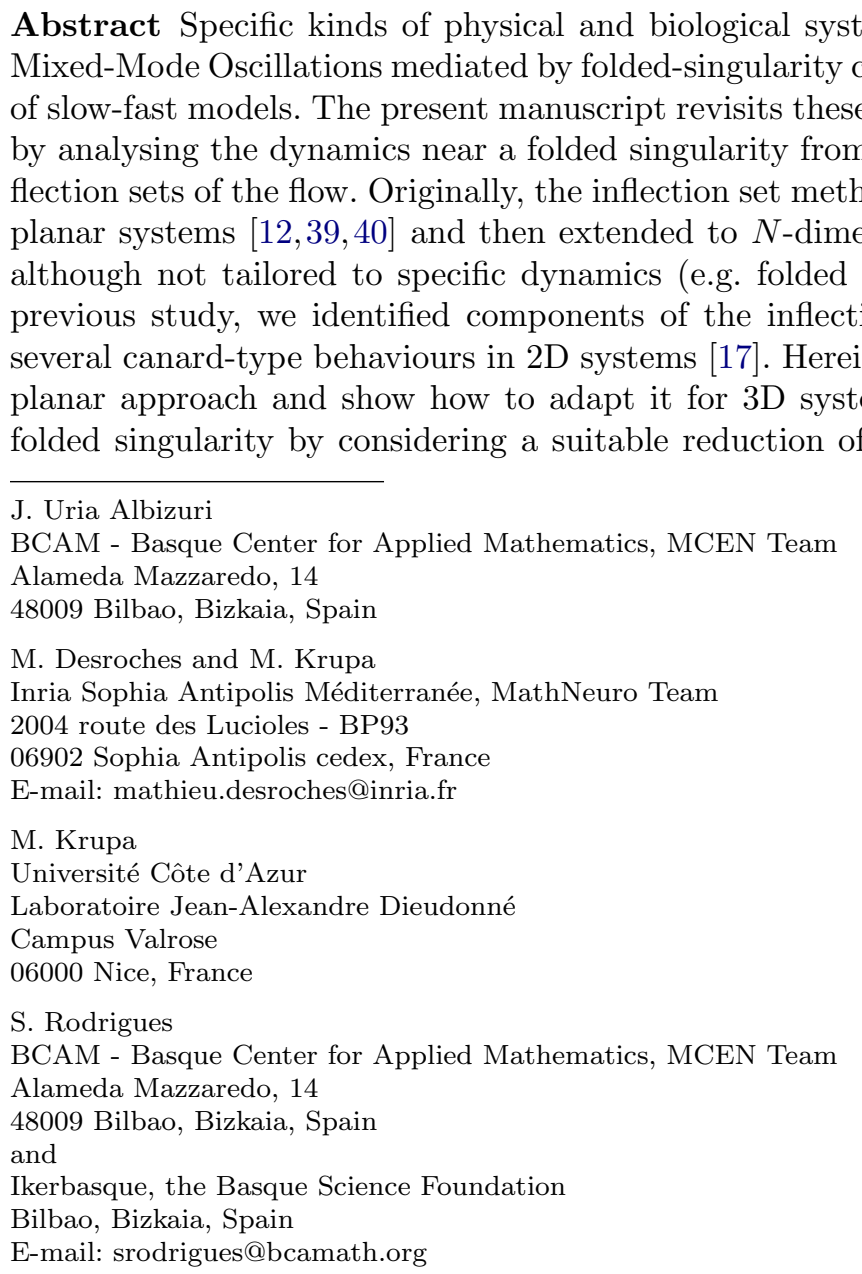


planar non-autonomous slow-fast systems. This leads us to the computation of parametrized families of inflection sets of one component of that planar (nonautonomous) system, in the vicinity of a folded node or of a folded saddle. We then show that a novel component of the inflection set emerges, which approximates and follows the axis of rotation of canards associated to folded-node and folded-saddle singularities. Finally, we show that a similar inflection-set component occurs in the vicinity of a delayed Hopf bifurcation, a scenario that can arise at the transition between folded node and folded saddle. These results are obtained in the context of a canonical model for folded-singularity canards and subsequently we show it is also applicable to complex slow-fast models. Specifically, we focus the application towards the self-coupled 3D FitzHugh-Nagumo model, but the method is generically applicable to higher-dimensional models with isolated folded singularities, for instance in conductance-based models and other physical-chemical systems.

Keywords Slow-fast systems · Canard solutions · Excitability · Inflection set $\cdot$ folded singularity $\cdot$ mixed-mode oscillations $\cdot$ threshold

\section{Introduction}

Spontaneous and complex oscillations are ubiquitous in open excitable multiscale (i.e. far from equilibrium) systems with dissipative structures, which are at the basis of living and many physical systems. The mechanisms that underpin these oscillations challenge both experimental and theoretical developments and a complete understanding remains elusive. This challenge can be appreciated for instance by realising that it was only until 1920s that Van der Pol exhibited the first multi-scale dynamical system with a dissipative structure, while previous mathematical theories had failed. Only from the 1980s some scientific and mathematical progress started to allow a full slow-fast understanding of such complex dynamics. Dissecting the mechanisms associated to these oscillations will lead to improved technologies that replicate these systems but with unwanted pathological states, for example via control. In the present manuscript, we first survey the inflection set method for planar systems $[12,15,17,23,39,40]$ and then show how to adapt it in order to enable the characterisation and classification of complex oscillations mediated by socalled canards [6,31], which are trajectories that are sensitive to both external inputs and to initial conditions despite not being associated with full-fledged chaotic behaviour.

Canard-mediated complex oscillations and dissipative structures are described by excitable multi-scale dynamics, typically expressed in the form of a singularly perturbed ordinary differential equation (ODE) that can be written in two equivalent ways as follows

$$
\begin{aligned}
& \varepsilon \dot{x}=f(x, y) \\
& \dot{y}=g(x, y) \text {, } \\
& \text { (1) } \stackrel{\varepsilon>0}{\Longleftrightarrow} \\
& \begin{array}{l}
x^{\prime}=f(x, y) \\
y^{\prime}=\varepsilon g(x, y),
\end{array}
\end{aligned}
$$


where $(x, y) \in \mathbb{R}^{n} \times \mathbb{R}^{m}, f$ and $g$ are smooth functions. The dot and the prime denote differentiation with respect to the slow $(t)$ and fast $(\tau=t / \varepsilon)$ time, respectively, $0<\varepsilon \ll 1$ being the small time constant explicitly added in order to induce a timescale separation between the variables $x$ and $y$. Observe that the two systems are equivalent as far as $\varepsilon \neq 0$. Such dynamical systems have been successful in modelling many complex phenomena (e.g. chemical reactions [11], neuronal activity [43], semiconductor lasers [33], material science [42], astrophysics [34], etc.) where the timing and ordering of various processes ensures correct functions. For example, in neuronal electrical activity, single ion channel proteins change between different conformations on the microsecond timescale. However, this conformation change is voltagedependent and stochastic and thus the opening probability occurs on a longer timescale. In contrast, the timescale of the membrane potential that controls the voltage-dependent ion channels is on the order of milliseconds. This mix of various timescales appears to give rise to sudden, often surprising, jumps in the state of biological and physical systems and, in general, to complex oscillations. An illustration of this point is the electrical voltage of a neuron in response to an input step current, which indeed induces transitions between slow fluctuations and fast sharp rises (action potentials) as shown in Fig. 1 panel (a).

A large body of theoretical developments have been made in Mathematics, Physics and Engineering to dissect the mechanisms associated with these complex dynamics. To name a few, this includes adiabatic theory (mostly developed in the realm of thermodynamics) [10], bifurcation theory [3], catastrophe theory [52], geometrical singular perturbation theory (Fenichel theory) [22, 29], geometric desingularization or blow-up method [20,31], matched asymptotics [21], nonstandard analysis [7], singularity theory [25]. These combined methods have been influential in describing canards, which are one of the fundamental dynamical objects that explain the aforementioned sudden (non trivial) "jumps" in the state of biological and physical systems. Indeed, these methods enable perturbative studies of the singular limit $\varepsilon=0$ of equations (1) and (2), where canard behaviour can be shown to appear, and then to show the persistence of canards for $\varepsilon>0$ small enough.

Surprisingly, a separate small body of research, initiated (to the best of our knowledge) by M. Okuda [39], considered a novel route to the study of excitability via the notion of inflection sets, yet without any links to canards. We now survey this approach as it then developed, that is, in the context of planar slow-fast systems. This approach focuses on loci of phase-space points where the system's trajectories have zero curvature, showing that these loci play an important role in understanding the overall dynamics of the system. In a general planar slow-fast system the inflection set equation is obtained by first writing down the so-called trajectory equation of system (1) or (2) defined by

$$
\frac{d y}{d x}=\frac{\varepsilon g(x, y)}{f(x, y)}
$$


and subsequently applying implicit differentiation with respect to $x$ assuming that the inflection condition holds, namely $d^{2} y / d x^{2}=0$. Finally, rearranging terms leads to the inflection set equation

$$
f\left(f_{x} g-f g_{x}\right)+\varepsilon g\left(f_{y} g-f g_{y}\right)=0,
$$

where $f_{x}$ (resp. $f_{y}$ ) denotes the partial derivative of $f$ with respect to $x$ (resp. $y$ ), and the same for $g_{x}$ and $g_{y}$. The solutions to equation (4) form the inflection set in the $(x, y)$ plane and herein we denote it by $I^{\varepsilon}$.

Past the initial results of Okuda, inflection-type methods re-emerged through the work of Peng et al. [40], who applied it to canard-explosive dynamics [11] in the context of chemical reactions. Specifically, they showed that a component of the inflection set (in 2D systems) allows to separate, in a loose sense, headless canard trajectories from canards with head (these will be explained in due course). However, they erroneously formulated a result claiming that canard explosions were discontinuous, which eventually was amended by Brøns \& Bar-Eli [12].

Similar approaches came later and include, in particular, the work of Ginoux/Rossetto [23], and Desroches/Jeffrey [15]. More recently, we took a step forward in re-interpreting via the inflection set the concept of excitability threshold i.e. a boundary between slow and fast transitions, as depicted by the neuronal time-series in Fig. 1 (a), which we had previously shown to be well approximated by so-called maximal canards [15,17]. Moreover, we understood that the inflection set can be expressed in the contest of singularity theory where a distinguished parameter as well as unfolding parameters, including $\varepsilon$, explain the geometry and topological shapes of the inflection sets and thus enables to distinguish between headless canards and canards with head via maximal canards (excitability threshold).

These results, summarised in Fig. 1 panels (b), (c), (d) were established under a simplified neuronal model (i.e. a 2D FitzHugh-Nagumo model), although they can be readily applied (via numerical treatment) to conductance-based models (e.g. Hodgkin-Huxley). Specifically, the FitzHugh-Nagumo model is well known to behave, for classical parameter values, as a type-II neuron whereby its spiking regime arises, upon continuous and monotonic variation of an applied current, through a Hopf bifurcation. Moreover, it admits a canard explosion along the branch of limit cycles born at this Hopf bifurcation; see Fig. 1 (a) where two such canard cycles are shown, one with head $\gamma_{\mathrm{h}}$ and one with head $\gamma_{\text {wh }}$. The same trajectories are plotted in phase plane (see panel (b)), which shows that both canards follow segments of the cubic critical manifold $S^{0}=\{f(x)=0\}$ (i.e. the fast nullcline, green curve in panel (c)) and have both stable and unstable trajectory segments. Moreover, the inflection set (traced in blue and superimposed onto the canard cycles in phase plane) shows two striking features. First, it is shown in panel (c) that there is a segment of the inflection set that approximates the unstable branch of the critical manifold thus capturing the excitability threshold and indeed maximal canards (i.e. canards that follow the entire unstable branch of the critical manifold). Second, the highlighted grey rectangle in panel (c) and its corresponding zoom 


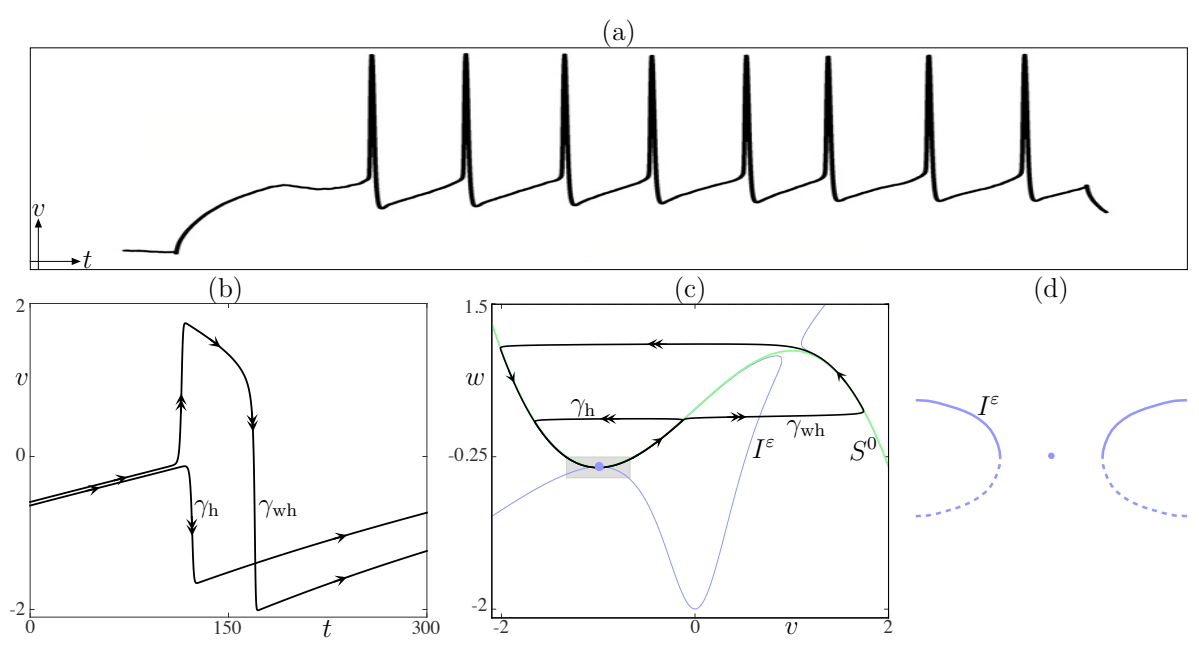

Fig. 1 Panel (a): spiking data from spiny neurons of rats' neostriatum, extracted from [30] ("Copyright 2004 Society for Neuroscience"). Panels (b) to (d): Inflection method in a canard-explosive system, namely the classical FitzHugh-Nagumo neuron model: $\varepsilon \dot{v}=v-$ $v^{3} / 3-w+I ; \quad \dot{w}=v+a-b w$. This is a type-II neuron, hence the excitability threshold is not well-defined, however it is best approximated by the repelling segment of the maximal canard, which can itself be approximated by one component of the inflection set $I^{\varepsilon}$ (blue in (c)). Here we show two canard cycles, one headless canard $\gamma_{\mathrm{h}}$ and one canard with head $\gamma_{\mathrm{wh}}$, for which we show their $v$ time profile ( in panel (b)) and their projection onto the $(v, w)$-phase plane, together with the critical manifold $S^{0}$ and the inflection set $I^{\varepsilon}$ (blue curves) in (c). In panel (d), we show a sketched zoom of the inflection set shown in (c), near the lower fold of $S^{0}$; it has three connected components: two are homeomorphic to parabolae and the equilibrium point (blue dot).

(panel (d)) show the key topological feature (in the sense of singularity theory), which delineates the boundary between sub-threshold headless canards $\left(\gamma_{h}\right)$ and super-threshold canards $\left(\gamma_{w h}\right)$, which effectively correspond to action potentials.

Our previous work highlighted the strengths of the inflection set method. Specifically, the inflection set approximates the Fenichel slow manifold [22] up to second order in $\varepsilon$, and thus explains the dynamics along the perturbation of normally hyperbolic compact subsets of the critical manifold. However, it also goes beyond in that it also characterises the behaviour along the nonnormally hyperbolic manifold region where Fenichel theorems fail. In the 2D case, this leads to the possibility of unfolding canards associated to the excitability threshold. More generally, curvature-based methods [23] and similar approaches such as the zero-derivative method [9] can approximate Fenichel slow manifolds to any order in $\varepsilon$ in general $N$-dimensional systems (even without explicit timescale separation). However, they require a substantial computational effort and they also produce many spurious solutions known as ghosts; see [9] for details. Moreover, these methods have not yet captured canard dynamics beyond van der Pol type canard explosions, for instance ro- 
tational dynamics near folded singularities (defined below) or the dynamics near delayed bifurcations.

The present manuscript proposes a different approach, entirely based upon the planar inflection set method which, as we show, can be adapted to tackle certain three-dimensional dynamics that can locally be reduced to planar dynamics with a drift. In particular, we will focus on systems with folded singularities and with certain types of mixed-mode oscillations (MMOs) [18], hence showcasing how the planar inflection set method can give information about a wider class of canard solutions.

MMOs are complex oscillations, whereby the time-series will have periods of small and large amplitude oscillations as shown in the experimental example of Fig. 2 as well as in the self-coupled FitzHugh-Nagumo model in Fig. 6 (b). In the case of periodic MMOs, the oscillatory patterns can be characterised by a pair of integers, namely the number of small and the number of large oscillations per period, which follow the Farey arithmetic [35]. In multipletimescale systems with of dimension at least 3, MMOs are typically induced by folded-singularity canards [18]. There are essentially four types of folded singularities, namely folded focus, folded node, folded saddle and folded saddle node. Herein we will focus on folded node and folded saddle as they are the most important cases near which robust MMOs can emerge and are relevant to applications.

Concretely, we consider a prototypical system given by an extension of (5) of the form:

$$
\begin{aligned}
& x^{\prime}=f(x, y) \\
& y^{\prime}=\varepsilon g(x, y, z) \\
& z^{\prime}=\varepsilon \mu
\end{aligned}
$$

where the $(x, y)$ subsystem has a canard explosion. In this system there is a folded node for $\mu>0$ and folded saddle for $\mu<0$. Since $z$ is only a slow drift, we can compute the inflection set of the $(x, y)$-system with forcing $z(t)$ using the planar inflection set method with an adaptation that takes into account this time-dependent forcing. We then show that this inflection set captures the qualitative features of the dynamics near a folded singularity. More specifically, the topological shape of the inflection set includes an additional closed component compared to the 2D case (where $z$ is constant), in the form of a bubble. We show that such bubble components of the inflection set not only characterise the emergence of canards but also, in the singular limit, follow (along time) the rotation axis associated with the folded node (weak singular canard) and with the folded saddle (faux singular canard). It is known [49] that near a folded node or folded saddle the dynamics can be, at lowest order, considered to be a canard explosion with a drift along the fold line. In other words, if the slow variables are represented as one that parametrizes the fold and one tranverse to the fold, then the inflection set of the variable transverse to the fold will in general give the same information as for system (5).

To best expose our findings, we will first consider a canonical 3D system displaying a folded node or a folded saddle. We then show that the framework 
is suited to applications by computing inflection sets on a well-known 3D excitable model, the so called self-coupled FitzHugh-Nagumo model $[14,50]$. We also apply the method to a 2 fast/1 slow system near a so-called delayed (or dynamics) Hopf bifurcation [4] where a family of inflection bubbles exists near the rotation axis of that system, which is consistent folded-singularity dynamics as a typical transition from a folded node to a folded saddle is often accompanied with slow passage through Hopf bifurcations [32].

Curvature-based methods, and more generally methods for approximating slow manifolds, have been developed for $N$-dimensional systems $[9,26,23]$. However, they have not been tailored to specific dynamics like those near folded singularities. We show here that, modulo a simple adaptation of the inflection set method for planar systems, allowing non-autonomous terms, one can capture key features of certain truly 3D systems, related to their foldedsingularity-induced rotation axes. This approach is not restricted to simple models and systems with explicit time scale separation (i.e. singularly perturbed systems) since it only relies on the differential geometric structure of the system's solutions. Hence it can be use for detection of folded node and folded saddle singularities in systems without explicit timescale separation, e.g. in the Hodgkin-Huxley model.

Methods associated to inflection sets go beyond what is presented here. In particular, in [45] we developed a pseudo-arclength continuation algorithm that detects inflection points of trajectories along solution branches. This computational tool was then applied to a neuronal model of absence seizure that explains $3 \mathrm{~Hz}$ (poly)spike-wave electrical discharges as observed in electroencephalogram (EEG) recordings $[44,46]$. The two key insights were: 1 . Different spike-wave patterns could be identified via inflections and related to a patients EEG seizure recordings across different seizure episodes. Detected infections could also be followed along a two-parameter bifurcation diagram, tracing out a patient-dependent signature pathway. 2 . The obtained pathway dictates whether or not a patient is responsive to anti-epileptic drugs [38]. Beyond neuroscience, the inflection set method could be applied in other areas, for instance in Gibbs thermodynamics, which can be related to the slow limit of adiabatic systems via the study of a contact geometrical structure [2].

The rest of the paper is organized as follows. In Section 2, we show the planar inflection set method can be easily adapted it in order to compute time-dependent inflection-lines in minimal folded-singularity models. We then study how they are related to special solutions of these systems, which are canard solutions and act as rotation axes. At the end of the section, we briefly show that the method can characterize rotation axis as well as trajectories contracting onto it and at a later time (and location in phase space) expand away from it, in a simplified model of delayed Hopf bifurcation. Then in Section 3 we apply this approach to a neuron model, namely the self-coupled FitzHughNagumo model. Finally, we draw conclusions and present a few perspectives of the present work. 


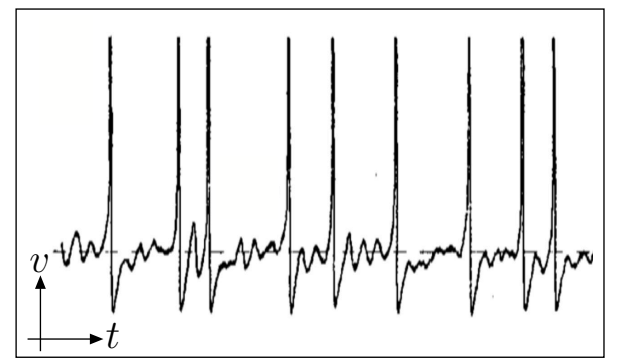

Fig. 2 Experimental mixed mode oscillations corresponding to a recording of dorsal root ganglion neurons of rats in vitro showing an irregular alternation between subthreshold oscillations and spikes, which can then termed experimental MMOs; extracted from [1] ("Copyright 2002 Society for Neuroscience").

\section{Inflection curves in 3D minimal systems with folded singularities}

2.1 The minimal folded-singularity system as a planar non-autonomous system

Here we consider a canonical three-dimensional system with two slow variables and an underlying folded singularity (either a folded node or a folded saddle) that induces complex oscillations. There are several theoretical machineries to understand the transitions mediated by folded singularities but herein we will show how the inflection curves of the flow of such a dynamical system can also unfold them. To best convey this methodology (in an intuitive way), we will dissect the slow-fast system and analyse the inflection set by simply following a succession of elementary calculations. However, the same problem can be cast in terms of singularity theory, which is mathematically more elegant; the key elements of this approach are provided in the Appendix A. To this end, we first apply this analysis to the following canonical folded-node model (expressed as a singularly perturbed ODE):

$$
\begin{aligned}
& x^{\prime}=-y+x^{2} \\
& y^{\prime}=\varepsilon(z+x) \\
& z^{\prime}=\varepsilon \mu,
\end{aligned}
$$

with $\mu \in \mathbb{R}$. System (6) is the fast-time system (fast time being denoted as $\tau$ ) where is $x$ is the fast variable, while $(y, z)$ are slow. The construction of this three-dimensional system (and indeed the mechanism behind its folded-node dynamics) is based on the idea of prescribing a slow dynamics on a parameter that unfolds a canard explosion of a planar slow-fast system that possesses a two-dimensional folded critical manifold. In this system, the prescription of the slow-dynamics is given by constant slow drift (i.e. this is the simplest case) and its sign determines whether the system is a minimal folded-node system or a minimal folded-saddle system. Following the standard analysis in slow-fast dynamics, time can be rescaled (posing $t=\varepsilon \tau$ ), which yields an equivalent 
system (for all $\varepsilon \neq 0$ ) in slow-time parametrization as follows

$$
\begin{aligned}
\varepsilon \dot{x} & =-y+x^{2} \\
\dot{y} & =z+x \\
\dot{z} & =\mu,
\end{aligned}
$$

where the overdot denotes $\frac{d}{d t}$. The singular limit $(\varepsilon=0)$ of the slow-time system (7) leads to the so-called slow subsystem described by the following differential-algebraic equation

$$
\begin{aligned}
& 0=-y+x^{2} \\
& \dot{y}=z+x \\
& \dot{z}=\mu .
\end{aligned}
$$

The slow flow is restricted to the critical manifold $S^{0}:=\left\{y=x^{2}\right\}$ and nowhere else. Since the fast dynamics is enslaved by the slow dynamics via the algebraic constraint $\left(0=-y+x^{2}\right)$ then we can recover the hidden fast-enslaved dynamics by implicit differentiation of the algebraic equation with respect to $t$. Further projecting the resulting system onto the $(x, z)$-plane reveals the slow subsystem

$$
\begin{aligned}
-2 x \dot{x} & =-z-x \\
\dot{z} & =\mu,
\end{aligned}
$$

Note that this system ceases to be defined for $x=0$, which corresponds to the fold curve (denoted by $F$ ) of the critical manifold $S^{0}$. A way to understand the slow flow up to $F$ involves introducing an auxiliary system, called the desingularised reduced system (DRS), obtained from system (9) by rescaling time by a factor $-2 x$. In this case, the DRS corresponds to the following system

$$
\begin{aligned}
& x^{\prime}=-z-x \\
& z^{\prime}=-2 \mu x .
\end{aligned}
$$

where here the prime denotes yet a new time, corresponding to the singular time rescaling mentioned above. This time rescaling introduces an equilibrium for the DRS at the origin and its stability can be computed. However, these computations have to take into account the algebraic constraint, in that the flow has to take place on the critical manifold. These computations lead to stability conditions that depend on $\mu$ through its eigenvalues $\lambda_{ \pm}$and associated eigenvectors $\mathbf{v}_{ \pm}$given by

$$
\begin{aligned}
& \lambda_{ \pm}=\frac{1}{2}(-1 \pm \sqrt{1+8 \mu}) \\
& \mathbf{v}_{ \pm}=\left(\begin{array}{c}
1 \\
-\frac{2 \mu}{\lambda_{ \pm}}
\end{array}\right) .
\end{aligned}
$$

Therefore the DRS (10) has at the origin a stable focus for $\mu<-1 / 8$, stable node for $-1 / 8<\mu<0$ and saddle for $\mu>0$. However, note that in the true 
slow subsystem (9), this point is not an equilibrium. What is more, the singular rescaling has the effect of reversing the direction of the flow of (9) along the repelling sheet of $S^{0}$ compared to the flow of the DRS. This analysis gives the insight as to why, in the singular limit, trajectories cross the origin in finite time. Indeed, these trajectories, termed singular canards, come along the attracting sheet of $S^{0}$ and continue along its repelling side past the origin (that is then denoted either a folded equilibrium or a folded singularity). Note that it is known that singular canards are only possible in the folded-node and in the folded-saddle cases, hence we shall restrict our analysis to these two scenarios. In the case of a folded saddle, there are two types of singular canards. The first is called singular true canard, which is associated to trajectories following the stable manifold of the saddle equilibrium of the DRS (10) (i.e. eigenvalue $\lambda_{-}$). Alternatively, it is related to trajectories flowing from the attracting sheet to the repelling sheet of $S^{0}$ via the origin in the slow flow (9). The second singular canard, is called singular faux ("false") canard, which crosses from the repelling to the attracting side of $S^{0}$ in (9) via the unstable manifold of the saddle of (10)(associated to the eigenvalue $\lambda_{+}$and its eigendirection). In the folded-node case, the strong and weak eigendirections of the node equilibrium of the DRS at the origin lead to existence of the strong singular canard (associated with the eigenvalue $\lambda_{-}$) and of the weak singular canard (associated with the eigenvalue $\lambda_{+}$) in the slow flow (9), respectively.

\subsection{Special solutions of the minimal system (7)}

Conveniently, although the minimal folded-singularity system (7) is nonlinear, it has the double peculiarity that, first, it admits explicit solutions for any $\varepsilon$ (even large) and, second, it has explicit canard solutions for small enough $\varepsilon$. This fortunate situation was first reported in [8]. In the slow-time parametrization, the explicit solutions are given by

$$
\begin{aligned}
& x_{\varepsilon}(t)=\frac{\alpha}{\varepsilon}\left(t-t_{0}\right) \\
& y_{\varepsilon}(t)=-\alpha+\frac{\alpha^{2}}{\varepsilon^{2}}\left(t-t_{0}\right)^{2} \\
& z_{\varepsilon}(t)=\mu\left(t-t_{0}\right),
\end{aligned}
$$

where $t$ is time, $t_{0}$ is the initial time and $\alpha$ satisfies the quadratic equation $2 \alpha^{2}-\varepsilon \alpha-\varepsilon^{2} \mu=0$. This quadratic equation in $\alpha$ ensures that (12) solves the differential system (7). Indeed, the $\dot{y}$ equation applied to (12) gives $2 \frac{\alpha^{2}}{\varepsilon^{2}}(t-$ $\left.t_{0}\right)=\mu\left(t-t_{0}\right)+\frac{\alpha}{\varepsilon}\left(t-t_{0}\right)$, which must be valid for any $t$, hence the quadratic equation in $\alpha$, obtained after rearrangement of terms. The solutions to this quadratic equation are

$$
\alpha_{ \pm}=\frac{\varepsilon}{4}(1 \pm \sqrt{1+8 \mu}) .
$$

It turns out that these two special solutions parameterise the two canards near folded-node or folded-saddle singularities (i.e. the strong/weak singular 
canards and the true/faux singular canards discussed in the previous section) and their corresponding trajectory solutions are expressed as follows

$$
\begin{aligned}
& x_{ \pm, \varepsilon}(t)=\left(\frac{1 \pm \sqrt{1+8 \mu}}{4}\right)\left(t-t_{0}\right) \\
& y_{ \pm, \varepsilon}(t)=\left(\frac{1 \pm \sqrt{1+8 \mu}}{4}\right)\left(-\varepsilon+\left(\frac{1 \pm \sqrt{1+8 \mu}}{4}\right)\left(t-t_{0}\right)^{2}\right) \\
& z_{ \pm, \varepsilon}(t)=\mu\left(t-t_{0}\right) .
\end{aligned}
$$

This shows that these special canard solutions given by (13) have polynomial growth and stay for all times at a distance of order $\alpha \sim \varepsilon$ (for $\mu$ up to $O(1)$ ) from the critical manifold $S^{0}$. It is straightforward to check that, in the singular limit $\varepsilon=0$, these special solutions converge to two curves traced on the critical manifold with a linear relationship between $x_{ \pm}$and $z_{ \pm}$(satisfied for any $\varepsilon$ ) that is given by $x_{ \pm} / z_{ \pm}=(1 \pm \sqrt{1+8 \mu}) /(4 \mu)=-\lambda_{\mp} /(2 \mu)$.

In other words, the special solution $\left(x_{ \pm, \varepsilon}(t), y_{ \pm, \varepsilon}(t), z_{ \pm, \varepsilon}(t)\right)$ converges in the singular limit towards the eigenline associated with the eigenvalue $\lambda_{\mp}$ of the DRS, traced on the critical manifold $S^{0}$. Specifically, two cases emerge:

1. The special solution $\left(x_{-, 0}(t), y_{-, 0}(t), z_{-, 0}(t)\right)$ corresponds to the singular weak canard for $-1 / 8<\mu<0$ (folded-node case) and to the singular faux canard for $\mu>0$ (folded-saddle case).

2. The special solution $\left(x_{+, 0}(t), y_{+, 0}(t), z_{+, 0}(t)\right)$ corresponds to the singular strong canard for $-1 / 8<\mu<0$ (folded-node case) and to the singular true canard for $\mu>0$ (folded-saddle case).

For small $\varepsilon>0$, the special solutions described above still correspond to special canards of the full system, namely the $\varepsilon$-perturbation of the singular canards that exist in both the folded-node and the folded-saddle case. Since the mid-1980s, it is known that in the folded-node case, the perturbation of the singular weak canard, which for the minimal system (7) corresponds to the special solution $\left(x_{-, \varepsilon}(t), y_{-, \varepsilon}(t), z_{-, \varepsilon}(t)\right)$, acts as an axis of rotation around which all nearby trajectories rotate as they are trapped in a funnel region near the folded node; see $[7,18,50]$.

Amongst these rotating trajectories there are additional canard solutions called secondary canards, which are the boundaries in phase space between regions that separate trajectories with a given number of small-amplitude oscillations around this rotation axis (see Fig. 6 panel (c)). The maximal number of small oscillations, which corresponds to the maximal number of rotation sectors (bounded by secondary canards) is a function of the eigenvalue ratio at the folded node (as an equilibrium of the DRS) and, hence, a function of $\mu$. More recently, it has been shown that there are also rotating trajectories and secondary canards near a folded saddle. However in that case the axis of rotation is the faux canard; see [36]. These results were independently shown in the context of piecewise-linear slow-fast systems in [19]. For the canonical system (7) that we are herein considering it is rather convenient that the axis of rotation correspond to the same special solution in both the folded node 
and the folded saddle case, namely $\left(x_{+, \varepsilon}(t), y_{+, \varepsilon}(t), z_{+, \varepsilon}(t)\right)$. We will subsequently show that this special canard solution acting as a rotation axis can be captured through an extension of the inflection curve method to system (7), which locally describes any system near a folded singularity. Therefore, the approach presented next reveals a geometrical link that pertains to all slowfast systems in the vicinity a folded node or a folded saddle, once represented within an appropriate coordinate system.

\subsection{Detection of inflection curves of the $y$-component}

In this Section, we will adapt the planar inflection set approach to systems with a folded singularity and show that a new component of the resulting inflection set explains the axis of rotations of folded-singularity canards. Such systems can be at first generally written as follows

$$
\begin{aligned}
\varepsilon \dot{x} & =f(x, y, z) \\
\dot{y} & =g(x, y, z) \\
\dot{z} & =h(x, y, z),
\end{aligned}
$$

with $f, g, h$ being smooth functions and the critical manifold $S^{0}=\{f=$ $0\}$ being a folded surface in $\mathbb{R}^{3}$. We will employ the same methodology as developed in our previous work $[15,17]$, which corresponds to compute loci of phase-space points where system trajectories have zero curvature in a wellchosen plane, but we will further extend it to capture complex oscillations mediated by folded singularities.

To this end, we compute the inflection set of the $y$ variable only, which allows to locate the rotation region near the one fold curve of $S^{0}$, in addition to approximating slow manifolds as in [17].

We first rewrite (14) using $x$ as an independent variable:

$$
\begin{aligned}
& \frac{d y}{d x}=\varepsilon \frac{g(x, y, z)}{f(x, y, z)} \\
& \frac{d z}{d x}=\varepsilon \frac{h(x, y, z)}{f(x, y, z)},
\end{aligned}
$$

subsequently differentiate the first equation from (15) with respect to $x$ and set $\frac{d^{2} y}{d x^{2}}$ to 0 (zero curvature condition in the $(x, y)$ plane), which leads to

$$
f\left(f_{x} g-f g_{x}\right)+\varepsilon\left(g\left(f_{y} g-f g_{y}+f_{z} h\right)-g_{z} h f\right)=0
$$

Equation (16) is effectively the inflection set equation for system (14) in the $(x, y)$-plane, which is an extended version of the inflection set equation (4) computed in planar systems as in [17]. Indeed, here we must take into account the fact that $z$ also has a dynamic evolution. It may seem simplistic to compute the inflection set equation only in the $(x, y)$ plane, however this approach allows 
to reveal key information about folded singularities and associated rotational dynamics taking place near them, as we next explain.

Systems with folded singularity are three-dimensional slow-fast systems with two slow variables, yet auspiciously, near the folded singularity these 3D systems can be cast (via a suitable change of variables) as a $2 \mathrm{D}$ dynamics with a drift. Namely, the dynamics of the slow variable parametrizing the fold can be assumed to be only a drift $(\dot{z}=\mu)$, which is why the minimal system (7) is pertinent $[18,49,50]$. In particular for system (7) the equivalent of (15) can be formed, from which one obtains the $y$ inflection set equation in the form

$$
-\frac{\varepsilon^{2}(z+x)^{2}}{h^{2}}+2 x \frac{\varepsilon(z+x)}{h}-\varepsilon^{2} \mu \frac{1}{h}-\varepsilon=0,
$$

where $h=-y+x^{2}$. Rearranging and simplifying results in a $z$-dependent quadratic inflection equation

$$
h^{2}+(\varepsilon \mu-2 x(z+x)) h+\varepsilon(z+x)^{2}=0,
$$

where the general solution is given by $h_{ \pm}(x)=(-\varepsilon \mu+2 x(z+x) \pm \sqrt{\Delta}) / 2$, with discriminant $\Delta=[\varepsilon \mu-2 x(z+x)]^{2}-4 \varepsilon(z+x)^{2}$. Note that $z=\mu t+z_{0}$, hence the above formulas can also be expressed in terms of $x$ and $t$.

\subsection{The "bubble": the central part of the inflection set}

For a range of $z$-values (to be determined in due course), the central connected component of the inflection set is homeomorphic to a circle, with two pairs of fold points; see figures 3(c) and 4(c) for an illustration of this new component computed in the minimal system (7) near a folded node and a folded saddle, respectively. The fold points of this bubble with respect to the vertical axis correspond to zeroes of the discriminant $\Delta$ of the inflection equation, giving $\varepsilon \mu-2 x(z+x)= \pm 2 \sqrt{\varepsilon}(z+x)$, where $z$ is assumed to range from negative to positive values. Rearranging leads to a quadratic equation which has the following solutions

$$
x_{ \pm}^{ \pm}(z, \varepsilon)=\frac{1}{2}\left(-(z \pm \sqrt{\varepsilon}) \pm \sqrt{(z \mp \sqrt{\varepsilon})^{2}+2 \varepsilon \mu}\right) .
$$

We first proceed by showing that the solutions to (19) correspond to the vertical fold points of the bubble. This amounts to analysing under which conditions the limit points of the bubble at $x_{+}^{+}$and $x_{-}^{-}$are equal, as subsequently expressed by

$$
-(z+\sqrt{\varepsilon})+\sqrt{(z-\sqrt{\varepsilon})^{2}+2 \varepsilon \mu}=-(z-\sqrt{\varepsilon})-\sqrt{(z+\sqrt{\varepsilon})^{2}+2 \varepsilon \mu} .
$$

Following algebraic computations, this yields

$$
2 \sqrt{\varepsilon}-\sqrt{(z-\sqrt{\varepsilon})^{2}+2 \varepsilon \mu}=\sqrt{(z+\sqrt{\varepsilon})^{2}+2 \varepsilon \mu},
$$


(a)

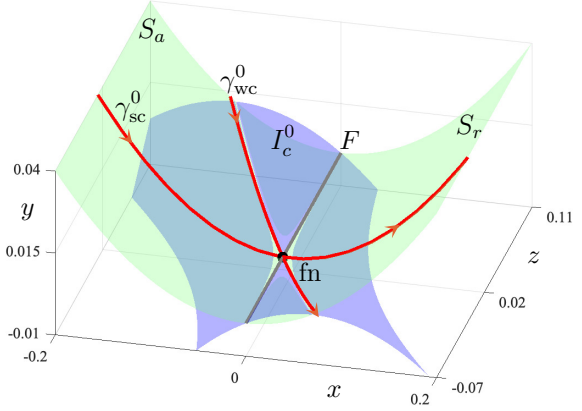

(b)

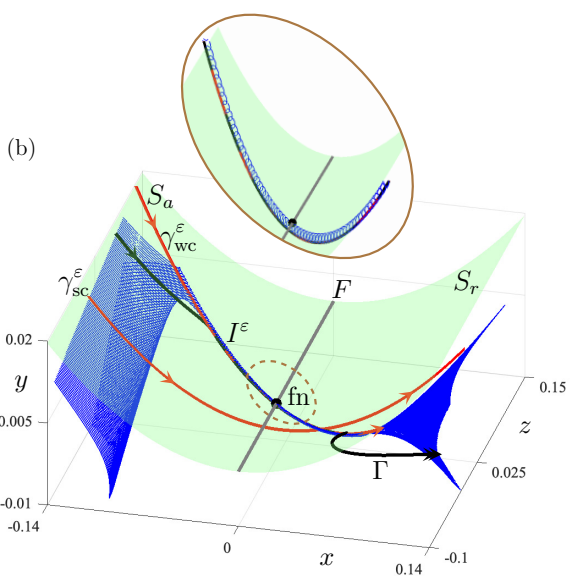

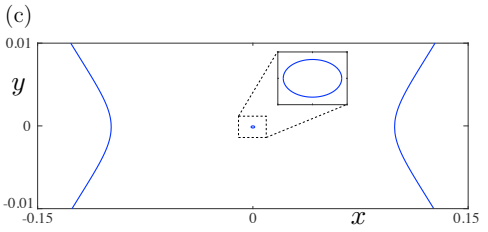

Fig. 3 Folded node case in system (7) with $\mu=-0.025$. (a) $\varepsilon=0$ : The green surface is the critical manifold $S^{0}$, the blue surface is the other (central) component solution of the inflection set equation at $\varepsilon=0(28), I_{c}^{0}$. Their two intersection curves are the fold $F$ (grey line) of $S^{0}$ and the singular weak canard $\gamma_{\mathrm{wc}}^{0}$ (red curve). The black dot indicates the folded node fn. (b) $\varepsilon=0.01$ : inflection surfaces are plotted in magenta. The rotation axis (magenta curve) is the weak canard special solution $\gamma_{\mathrm{wc}}^{\varepsilon}:=\left(x_{-, \varepsilon}(t), y_{-, \varepsilon}(t), z_{-, \varepsilon}(t)\right)$, lies inside the central surface, which is made of the bubbles in every corresponding time frame. The black dot indicates the folded node $\mathrm{fn}$, the black curve $\Gamma$ is a solution segment with small-amplitude oscillations about the rotation axis (weak canard), and the other red curve is the other special solution (strong canard) $\gamma_{\mathrm{sc}}^{\varepsilon}:=\left(x_{+, \varepsilon}(t), y_{+, \varepsilon}(t), z_{+, \varepsilon}(t)\right)$. The inset shows a zoom near fn (corresponding to the dashed ellipse in the main panel). (c) Intersection of the inflection surfaces with $\{z=0\}$, highlighting the middle component (bubble); compare with Fig. 9 (b) obtained via singularity theory.

which can be equivalently written as follows

$$
\varepsilon-z \sqrt{\varepsilon}-\sqrt{\varepsilon} \sqrt{(z-\sqrt{\varepsilon})^{2}+2 \varepsilon \mu}=0 .
$$

Simplification by $\sqrt{\varepsilon}$, squaring and reducing, yields $2 \varepsilon \mu=0$. This shows that the bubble shrinks to a single point only in the singular limit $\varepsilon=0$ or when $\mu=0$, thus recovering the results of the planar problem studied in [17] where indeed the bubble component of the inflection set is reduced to a single point (see also Fig. 1). Moreover, it can be easily shown that the only other possible merging of two points, amongst the four fold points $x_{+}^{ \pm}$is either $x_{+}^{+}$with $x_{+}^{-}$or $x_{-}^{-}$with $x_{-}^{+}$. The two cases can be treated simultaneously by solving $x_{+}^{+}=x_{+}^{-}$ and $x_{-}^{-}=x_{-}^{+}$, that is

$$
-(z+\sqrt{\varepsilon})+\sqrt{(z-\sqrt{\varepsilon})^{2}+2 \varepsilon \mu}=-(z+\sqrt{\varepsilon})-\sqrt{(z-\sqrt{\varepsilon})^{2}+2 \varepsilon \mu},
$$


(a)

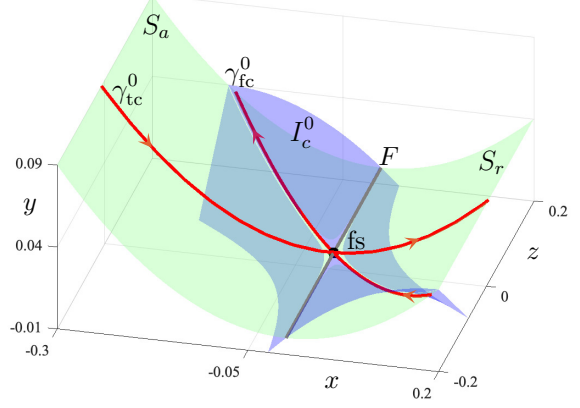

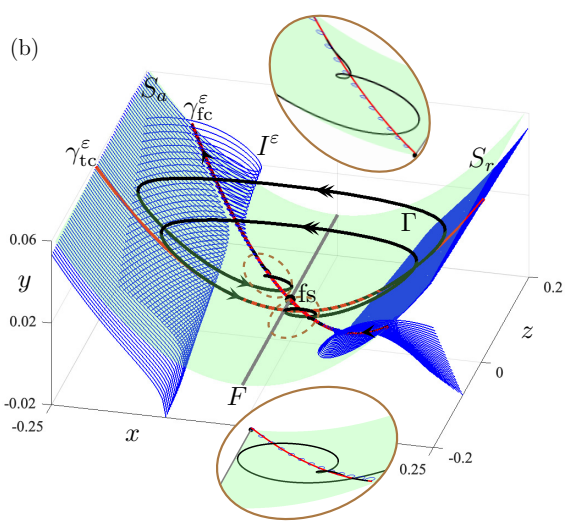

(b)

(c)

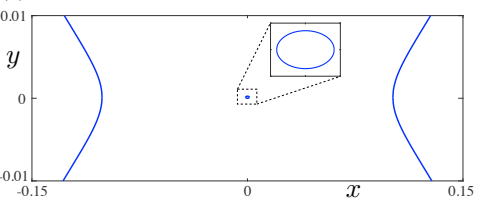

Fig. 4 Folded saddle case in system (7) with $\mu=0.025$. (a) $\varepsilon=0$ : The green surface is the critical manifold $S^{0}$, the blue one is the other (central) component solution of the inflection set equation at $\varepsilon=0(28), I_{c}^{0}$. Their two intersection curves are the fold $F$ (grey line) of $S^{0}$ and the singular faux canard $\gamma_{f_{c}}^{0}$ (red curve). The black dot indicates the folded saddle fs. (b) $\varepsilon=0.01$ : inflection surfaces are plotted in blue. The rotation axis (red curve) is the faux canard special solution $\gamma_{\mathrm{f}_{c}}^{\varepsilon}:=\left(x_{-, \varepsilon}(t), y_{-, \varepsilon}(t), z_{-, \varepsilon}(t)\right)$, lies inside the central surface, which is made of the bubbles in every corresponding time frame. The black dot indicates the folded saddle fs, the black curve $\Gamma$ is a solution segment with small-amplitude oscillations about the rotation axis (faux canard). Finally, the other red curve is the other special solution (true canard) $\gamma_{\mathrm{tc}}^{\varepsilon}:=\left(x_{+, \varepsilon}(t), y_{+, \varepsilon}(t), z_{+, \varepsilon}(t)\right)$. The insets show zooms near fs (corresponding to the dashed ellipses in the main panel). (c) Intersection of the inflection surfaces with $\{z=0\}$, highlighting the middle component (bubble); compare with Fig. 9 (b) obtained via singularity theory.

and

$$
-(z-\sqrt{\varepsilon})-\sqrt{(z+\sqrt{\varepsilon})^{2}+2 \varepsilon \mu}=-(z-\sqrt{\varepsilon})+\sqrt{(z+\sqrt{\varepsilon})^{2}+2 \varepsilon \mu},
$$

respectively. Both these equations identically reduce to $(z \mp \sqrt{\varepsilon})^{2}+2 \varepsilon \mu=0$, which only has solutions (for $\varepsilon \neq 0$ ) and $\mu<0$, that is, in the folded node scenario. This indicates that, in the folded saddle case, the bubble persists for all times and only shrinks as $\varepsilon$ or $\mu$ tends to 0 . Besides, for $z=0$, the bubble does exist and the four fold points $x_{ \pm}^{ \pm}$are clearly distinct (for $\varepsilon \mu \neq 0$ ). Consequently, to determine the $z$-span of the bubble component, one must seek the smallest $z$-value (in absolute value) such that two of these four fold points coincide, hence satisfying (24). This occurs at

$$
\begin{aligned}
& z_{+}=\sqrt{-2 \varepsilon \mu}-\sqrt{\varepsilon}>0 \\
& z_{-}=-z_{+}<0
\end{aligned}
$$


As $\varepsilon \rightarrow 0$, the four $z$-dependent fold points of the inflection set $x_{ \pm}^{ \pm}(\varepsilon, z)$ converge to

$$
\lim _{\varepsilon=0} x_{ \pm}^{ \pm}(\varepsilon, z)=\frac{1}{2}(-z \pm|z|),
$$

which leads to two solutions $x=0$ and $x=-z$. The first one corresponds to the fold line $F$ of the critical manifold $S^{0}$ and the second one to the singular limit of the rotation axis, as we verify below.

To briefly summarise, we compute the inflection set of the minimal foldedsingularity 3D system (7) by first recasting it into a 2D non-autonomous system (15) and subsequently computing the inflection set at every $z$ using the method developed in our previous study [17]. We then show that there exists an interval of values of $z$ for which this inflection set has, near the fold of the critical manifold, an additional component shaped like a bubble. For every small enough $\varepsilon>0$ and every $z \in\left(z_{-}, z_{+}\right)$the family of time-dependent bubbles surrounds the intersection point between the axis of rotation of the original system (7) and the cross-section $\left\{z=z_{0}\right\}$; see Figs 3 (b) and 4 (b). Indeed, at $z=0$, the fold points that bound the bubble, $x_{+}^{+}(0, \varepsilon)$ and $x_{-}^{-}(0, \varepsilon)$, are given by

$$
\begin{aligned}
& x_{+}^{+}(0, \varepsilon)=\frac{\sqrt{\varepsilon}}{2}(\sqrt{1+2 \mu}-1) \\
& x_{-}^{-}(0, \varepsilon)=-x_{+}^{+}(0, \varepsilon),
\end{aligned}
$$

when the $x$-coordinate of the rotation axis is equal to 0 . Therefore, at $z=0$, the rotation axis has its $x$-coordinate strictly located in between those of the fold points of the bubble. By continuity, this persists for a (possibly small) interval of $z$-values that contains 0 . On the other hand, a simple calculation shows that, for $z=0$, the bubble is symmetric with respect to the $x$-axis and the maximum and minimum $y$-coordinates along the bubble hence occurs at $x=0$ where they are equal to 0 and $\varepsilon \mu$, respectively. However, the $y$-coordinate of the rotation axis point corresponding to $z=0$ is equal to

$$
-\left(\frac{1 \pm \sqrt{1+8 \mu}}{4}\right) \varepsilon \approx\left(\mu-2 \mu^{2}+o\left(\mu^{2}\right)\right) \varepsilon,
$$

for $\mu \rightarrow 0$. This shows that the rotation axis, in the $t=0$-frame, lies inside the bubble in the folded-saddle case $\mu>0$ (small enough) and slightly outside of it in the folded-node case $\mu<0$ (small enough). In the $\varepsilon \rightarrow 0$ limit, both the rotation axis and the bubble converge to the singular rotation axis as aforementioned.

In the limit $\varepsilon \rightarrow 0$, this bubble converges to the singular limit of the axis of rotation, that is, the singular weak canard for $-1 / 8<\mu<0$ and the singular faux canard for $\mu>0$, for $\mu$ small enough. Moreover, the limit for $\varepsilon \rightarrow 0$ of the inflection set gives two quadratic surfaces, namely the critical manifold and another parabolic cylinder. These surfaces intersect along two curves, the fold of the critical manifold $F$ and the singular limit of the rotation axis $\left(\gamma_{w c}^{0}\right)$ and 
these simultaneously intersect at the folded singularity (fn). This is evident, since the $\varepsilon=0$ limit of the inflection set equation (18) reduces to

$$
h^{2}-2 x(z+x) h=0
$$

which results in two solutions, namely $h=0$ that corresponds to the critical manifold $S^{0}$, and $h=2 x(z+x)$. Thus, there are two quadratic surfaces that intersect along two curves, namely $x=0$, corresponding to the fold curve of $S^{0}$, and the curve defined by $x=-z(x(t)=-\mu t)$ and $y=x^{2}$, respectively. Similarly, the singular limit of the rotation axis (as explained above) correspond to the curve $\left(x_{-, 0}(t), y_{-, 0}(t), z_{-, 0}(t)\right)$ defined by

$$
\begin{aligned}
& x_{-, 0}(t)=\left(\frac{1 \pm \sqrt{1+8 \mu}}{4}\right) t \\
& y_{-, 0}(t)=x_{-, 0}(t)^{2} \\
& z_{-, 0}(t)=z
\end{aligned}
$$

where the following holds

$$
\frac{x_{-, 0}}{z_{-, 0}}=\frac{1-\sqrt{1+8 \mu}}{4 \mu} \approx-\mu t, \quad \mu \rightarrow 0 .
$$

This reveals that for sufficiently small $\mu$ (negative or positive), one component of the intersection of the two inflection surfaces is the singular rotation axis $\gamma_{w c}^{0}$, and the other component is the fold line $F$ of $S^{0}$. Moreover, both components intersect at the folded singularity (node or saddle) located at the origin (fn). Figures 3 (a) and 4 (a) illustrate this finding. In the limit for $\mu \rightarrow 0$, we recover the result of our previous study, on the analysis of inflection set of the 2D $(x, y)$ problem with $z$ being a parameter [17]. Indeed, in the limit $\mu=0$, the inflection equation (17) converges to

$$
-\left(\frac{d y}{d x}\right)^{2}+2 x \frac{d y}{d x}-\varepsilon=0,
$$

which is precisely the inflection equation for the planar system

$$
\begin{aligned}
\varepsilon \dot{x} & =-y+x^{2} \\
\dot{y} & =z+x,
\end{aligned}
$$

where the drift speed $\mu$ of the second slow variable $z$ of the minimal foldedsingularity system (7) converges to 0 and thus $z$ becomes a parameter of the planar system (31). To re-iterate, this explains why the bubble component of the inflection set of system (7) shrinks to a point as either $\varepsilon$ or $\mu$ tends to 0 . In the planar system (31) this point corresponds to the equilibrium, which has to lie on the inflection set by definition. In conclusion, the passage from the planar slow-fast system (31) to the 3D minimal folded-singularity system (7) through the add-on of a dynamics of constant speed $\mu$ for $z$, is felt in a similar way at the level of the inflection sets in both systems. 


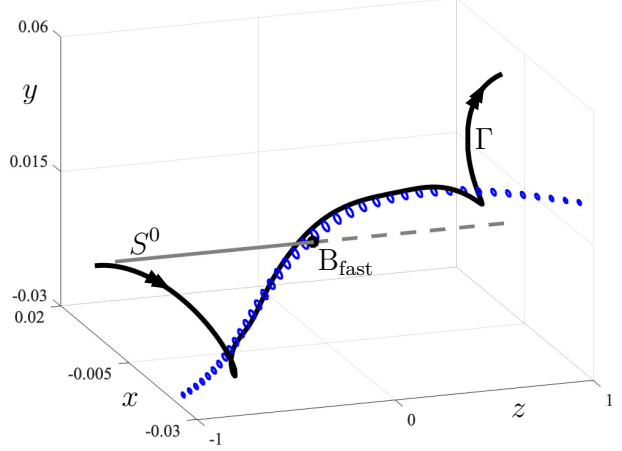

Fig. 5 Family of $y$ inflection curves in the simplified Shishkova system (32) for $\varepsilon=0.05$ and $\alpha=1$. This shows that the 3D extension of the method inflection method also works in the case of delayed-Hopf bifurcation systems and, more generally, in 2 fast / 1 slow systems.

\subsection{Inflection tube near delayed bifurcation points}

As was seen before, there is a new solution component to the inflection equation near a folded node or a folded saddle. At the transition between the two, there is the folded saddle-node scenario of type II, which is known to be very closely related to a delayed passage through a Hopf bifurcation [32]. This latter phenomenon has been widely studied both mathematically and within the context of numerous application area, since its discovery by Shishkova in the mid 1970s [48] and the complete theoretical proof by Neishtadt about a decade later [37]. In order to explore the link between minimal systems with folded singularities and systems undergoing delayed passage through a Hopf bifurcation, we consider a simplified scenario for the delayed passage in a linear where a stable focus of the fast subsystem loses stability and becomes an unstable focus. This simpler system is referred to as the simplified Shishkova model [27] and its equations read

$$
\begin{aligned}
& x^{\prime}=z x-y+\varepsilon h(z) \\
& y^{\prime}=z y+x \\
& z^{\prime}=\varepsilon,
\end{aligned}
$$

where we choose $h(z)=\alpha z$ with $\alpha>0$. Let us compute the $y$-inflection curves of system (32). Proceeding as before, one can write the $z$-dependent trajectory equation in the $(x, y)$ plane and, after simplification, the $z$-dependent $y$-inflection curve equation, which takes the form

$$
\left(\frac{d y}{d x}\right)^{2}-\frac{d z}{d x}(x+\varepsilon \alpha) \frac{d y}{d x}+\frac{d z}{d x} y+1=0 .
$$

Therefore, after some rearrangement and substitutions for $d y / d x$ and $d z / d x$, we obtain an implicit equation which we rewrite, after twice completing a 
square, as

$$
\left(z y+x\left(1-\frac{\varepsilon}{2}\right)-\frac{\varepsilon^{2} \alpha}{2}\right)^{2}+\left(z(x+\varepsilon \alpha)-y\left(1-\frac{\varepsilon}{2}\right)\right)^{2}-K^{2}=0
$$

with: $K=\frac{\varepsilon}{2} \sqrt{(x+\varepsilon \alpha)^{2}+y^{2}}$. One can show that the solution to equation (34) corresponds to a bubble (i.e. an ellipse) for $z$ close enough to 0. In Fig. 5, we show this family of bubbles for $-1<z<1$ together with the family of equilibria of the fast subsystem of (32), which corresponds to the critical manifold $S^{0}$. A bifurcation in the fast subsystem, labelled $\mathrm{B}_{\text {fast }}$, occurs at $z=0$ and destabilizes the stable focus equilibrium, leading, in the full system, to a delayed passage through this bifurcation, which can be observed on the trajectory $\Gamma$ traced in Fig. 5. It is also clear from the figure that, for $z$-values that are close enough to the bifurcation $B_{\text {fast }}$, the trajectory of the full system stays close to the corresponding family of inflection bubbles. This behaviour is certainly comparable to what happens to $y$-inflection curves near a folded node and a folded saddle, as illustrated in Figures 3 and 4, respectively.

\section{Application to neural dynamics: the self-coupled FitzHugh-Nagumo model.}

The insights of the previous sections leads us to now consider the application towards a more realistic system, in particular, a neuron model that displays mixed-mode oscillations (i.e. transitions between subthreshold oscillations and spiking). Specifically, we consider a version of the FitzHugh-Nagumo neuron model with an autapse (i.e. a self-coupled neuron) that was proposed to explain experimental observations of neuronal oscillations that undergo frequency decrease despite of increased excitation within a neural tissue. The model is

(a)

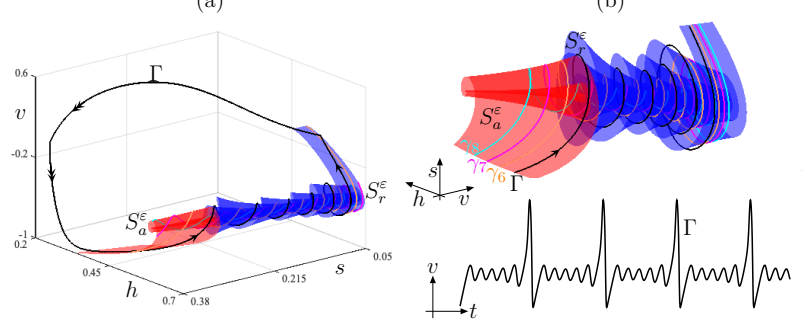

(c)

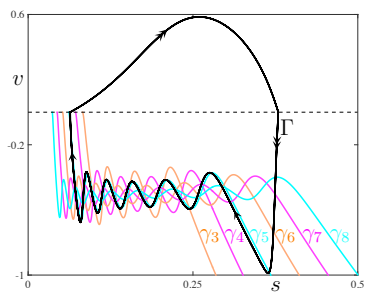

Fig. 6 Motivation to extend the inflection method to 3D folded-singularity model. We show a mixed-mode oscillation $\Gamma$ from the self-coupled FitzHugh-Nagumo model [14], both in 3D phase space (panel (a) and panel (b) top for a zoom) and in time for the $v$-variable (panel(b) bottom). Also shown in panel (a) are attracting $S_{a}^{\varepsilon}$ and repelling $S_{r}^{\varepsilon}$ slow manifold computed in the vicinity of a folded node. The slow manifold rotate around each other and intersect transversally along secondary canards $\gamma_{3-8}$ (colored curves), which we also show in projection onto the $(s, v)$-plane in panel (c). Each secondary canard acts as a threshold between MMO with $n$ and $n+1$ small-amplitude oscillations. 
described as follows

$$
\begin{aligned}
v^{\prime} & =h-0.5\left(v^{3}-v+1+s v\right) \\
h^{\prime} & =\varepsilon(2 h+2.6 v) \\
s^{\prime} & =\beta H(v)(1-s)-\varepsilon \delta s,
\end{aligned}
$$

where $H$ is the Heaviside function and $(\beta, \delta)$ are important systems parameters controlling the shape of the solutions. The $v$ is the voltage, $h$ is the gating variable (i.e. has the same role as the variable $w$ in the 2D FitzHugh-Nagumo model) and $s$ describes the synaptic activations. Note that the activation of the synapse is via a non-smooth activation $(H)$, which implies that an inactive synapse leads to the synaptic activity only being governed by the term $\varepsilon \delta s$. That is, below threshold (i.e. sub-threshold), the synaptic activity is simply proportional to $\varepsilon$, and thus overall, system (35) is a one fast / two slow smooth system, which can be shown to possess a folded singularity and canards that organise mixed-mode oscillations (MMOs) $[14,50]$ (see Fig. 6 panels (a) and (b)). The parameter $\beta$ is the strength of activation of synaptic activity, which ultimately controls the number of sub-threshold oscillations. This is achieved by the fact that synaptic strength has the effect of perturbing the superthreshold spiking activity towards a slightly different trajectory in phase space, and due to self neuronal stimulation, this spiking trajectory falls onto different sectors of rotation that control the number of sub-threshold oscillations (see Fig. 6 (c)). These sectors of rotation are mediated by secondary canards, which delineate the boundaries between different number of sub-threshold oscillations (see coloured lines in see Fig. 6 (c)). Interestingly, depending on the sign of $\delta$, the folded singularity can be a folded node $(\delta>0)$ or a folded saddle $(\delta<0)$; this is not obvious from equations (35) but can be obtained by computing

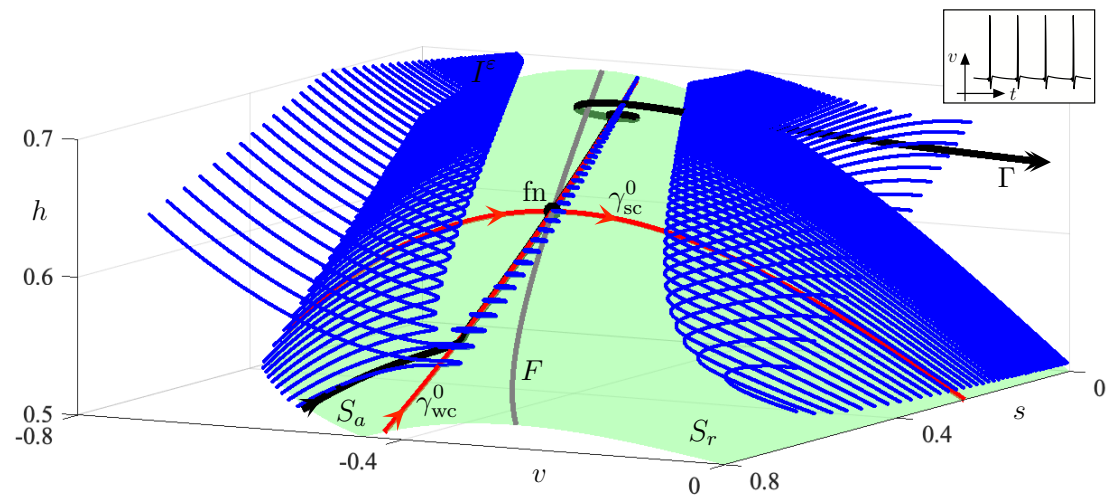

Fig. 7 Folded-node case for system (35) with $\varepsilon=0.003, \beta=0.036$ and $\delta=0.565$. Shown are the critical manifold $S^{0}$ (green surface), a selection of time-dependent inflection curves (blue) forming the inflection set $I^{\varepsilon}$, a MMO solution $\Gamma$ (black curve) that contains smallamplitude oscillations near the folded node $\mathrm{fn}$, twisting around the singular weak canard $\gamma_{\mathrm{wc}}^{0}(\mathrm{red})$, which is the axis of rotation in this context. The inset shows the $v$ time profile of $\Gamma$, which is indeed a periodic MMO. 


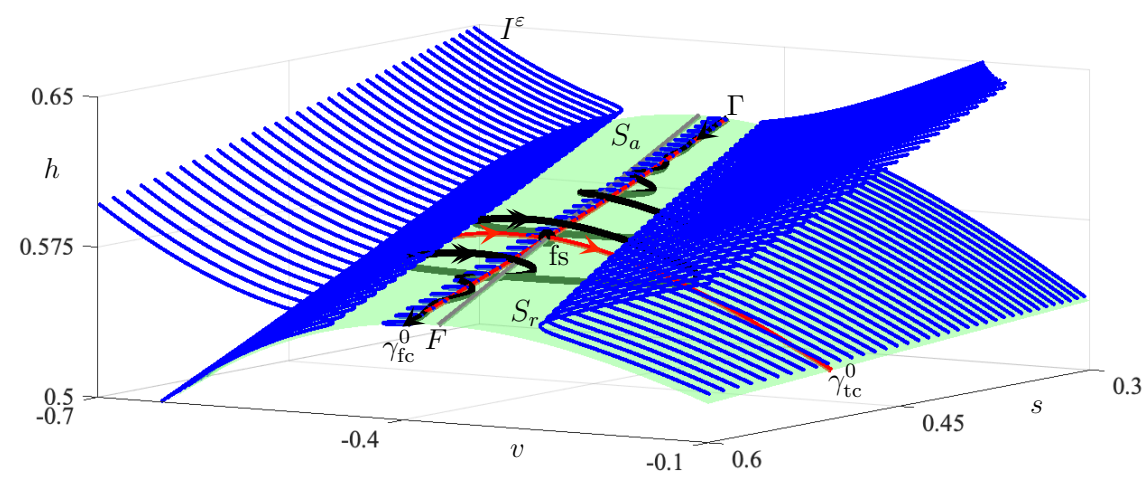

Fig. 8 Folded-saddle case for system (35) with $\varepsilon=0.001, \beta=0.036$ and $\delta=-0.565$. Shown are the critical manifold $S^{0}$ (green surface), a selection of time-dependent inflection curves (blue) forming the inflection set $I^{\varepsilon}$, a solution $\Gamma$ that contains small-amplitude oscillations near the folded saddle fs, twisting around the singular faux canard $\gamma_{\mathrm{fc}}^{0}(\mathrm{red})$, which is the axis of rotation in this context.

the associated DRS, see $[14,50]$ for details. Following the same procedure as outlined in previous sections, one can show that the time-dependent inflection set of system (35) captures the axis of rotation (weak canard in the foldednode case, faux canard in the folded-saddle case) of the sub-threshold small amplitude oscillations within this 3D FitzHugh-Nagumo neuron model.

To facilitate, we numerically solve this inflection equation (not shown) for both the folded saddle and the folded node case, and in both cases we fix $\beta=0.036$. Fixing $\delta=0.565$ results into a folded node, thus the overall system generates limit cycles containing small-amplitude oscillations near the folded node as well as large-amplitude oscillations corresponding to spikes, that is, MMOs. Further superimposing the computed inflection set as shown in Fig. 7 this confirms the observation made for the minimal folded-singularity system, namely that the bubble component of the time-dependent inflection set "traces out" the axis of rotation of the folded node cases for $\varepsilon>0$ sufficiently small, which corresponds to the weak canard. Equally, fixing $\delta=-0.565$ leads to the folded-saddle case, where trajectories may contain small oscillations near the faux canard acting as rotation axis. Moreover we compute the inflection, which is illustrated in Figs. 8. Here as well, we recover the analysis done in the minimal system, whereby the bubble component of the time-dependent inflection set "traces out", for $\varepsilon>0$ sufficiently small, the axis of rotation of the folded saddle, that is, the faux canard.

Note that the analysis and computations done on the example of the selfcoupled FitzHugh-Nagumo model, works equally well in any three-dimensional biophysical neuron model possessing a folded-node or a folded-saddle singularity. In particular, one can think of the three-dimensional reduction of the Hodgkin-Huxley (HH) model proposed in [43] where a folded node was shown to arise once a timescale separation parameter was postulated. This model 
has a very similar geometry as system (35) and it displays mixed-mode oscillations due to the presence of the folded node. Therefore, one expects to find the same inflection set structure in this 3D HH, in particular the bubble component following the rotation axis, even though the equation are substantially more complicated than that of system (35).

Finally, another interesting direction for future work will be to compute the inflection set structure in parabolic bursters. Indeed, we have shown in a previous work [16] that most parabolic bursting models, both biophysical (such as the Plant model [47]) and phenomenological (such as theta-like models), possess a folded-saddle singularity and that associated canard solutions organise spike-adding transitions responsible for the appearance of the bursting regime upon parameter variation. Therefore, it will be interesting to compute inflection sets near folded-saddle singularity of parabolic bursters and further analyse the role of the faux canard as a rotation axis in this bursting context. More generally, the information carried by inflection sets in the spike-adding regimes of various bursting models is an application of the ideas presented here that could shed further light onto such intricate dynamics, which have not yet been fully unravelled. In particular, we anticipate that the approach proposed here can be used to further analyse different types of complex oscillations generated by folded singularities and associated canard solutions, that is, not only mixed-mode oscillations but also certain bursting oscillations and possibly more complex combinations of them.

\section{Conclusion and perspective}

This work extends the inflection set method for a class of 3D slow-fast systems with two slow variables near a folded singularity of node or saddle type. To achieve this, we conveniently use the fact that such systems, at least in the vicinity of the folded singularity can be reduced to non-autonomous planar slow-fast systems where the second slow variable acts as a drift (akin to a time variable). This enables us to compute time-dependent inflection curves and show that in comparison to the planar case considered in our previous studies [17] the inflection set possesses one more connected component. This component (computed for a suitable time range) is able to approximate and track the rotation axis of canards near a folded node and near a folded saddle.

Moreover, maximal secondary canards (near a folded node) and maximal secondary faux canards (near a folded saddles) form boundaries between solutions with different oscillatory profiles, in particular with different numbers of subthreshold oscillations. Therefore, it is natural that these transitions, i.e. where a small-amplitude oscillation develops into a large-amplitude oscillation and, hence, acquire inflection points, relate to an inflection-set component. In the limit where $\varepsilon \rightarrow 0$, such trajectory segments with small oscillations converge towards the respective singular rotation axis. Consequently, the fact that near a folded node or a folded saddle, the inflection set contains one component that stays close to the rotation axis and converges to its singular limit, is to be 
expected. Yet, this fact was not been previously noticed and analysed in our previous work. Besides, while folded-node-induced MMOs have been heavily studied in the past 15 years or so and their associated dynamics is now quite well understood, solutions with small-oscillations near a folded saddle have only been discovered and analysed recently $[19,36]$.

Therefore, it is remarkable that we can capture these behaviour with inflection type methods and this opens further avenue of research to better understanding their possible role in excitable models. We show that this method can be used to detect folded-singularity canards in application that feature MMOs trajectories. To show case aspects of this, we apply it to the self-coupled FitzHugh-Nagumo. Noteworthy, the method could in principle be applied to higher-dimensional systems that have isolated folded singularities, following theoretical results from e.g. $[13,51]$ on $N$-dimensional slow-fast systems showing how to reduce to one fast and two slow variables locally near such singularities. Such study would reveal the funnel structure near a non-hyperbolic slow manifold in this local reduction to one fast and two slow dimensions.

A number of open questions emerge from the present work. First, although we have shown how to adapt the inflection set in the case of folded singularity to singularity theory (see appendix), a fully rigorous singularity-theoretic treatment of our results goes beyond the present paper. Second, future work should consider the folded-saddle-node scenario and the geometry of the inflection set near a singular Hopf bifurcation. Third, looking at oscillators with slow external forcing using the approach proposed here could help shedding further light on coupled neuron models and allow to explore their synchronization patterns from a geometrical view point. The same approach could be used to study adiabatic systems in thermodynamics. Forth, the present manuscript provides a descriptive study of the inflection set but it will be convenient that future studies establish theorems that could provide novel proofs of the existence and dissect the dynamics of singularity-folded canards.

On a longer-term horizon, we envisage a couple of interesting avenues. First, it would be challenging yet fruitful to combine the approach proposed in the present work with approaches based on Riemannian differential geometric tools that make full use of metric tensor [28] to study non-hyperbolic slow manifolds. Second, it would be worthy to investigate higher-dimensional systems with complex oscillatory behaviour from the inflection set viewpoint. Indeed, provided the configuration and geometry of slow and fast modes of a given high-dimensional system allows for the presence of isolated folded singularities, then the approach herein proposed could in principle be applied. In such a situation, this approach could potentially help to understand the lowdimensional dynamics of a high-dimensional system near non-hyperbolic slow manifold by finding the skeleton of funnels of rotating motion in these regions of phase space. 

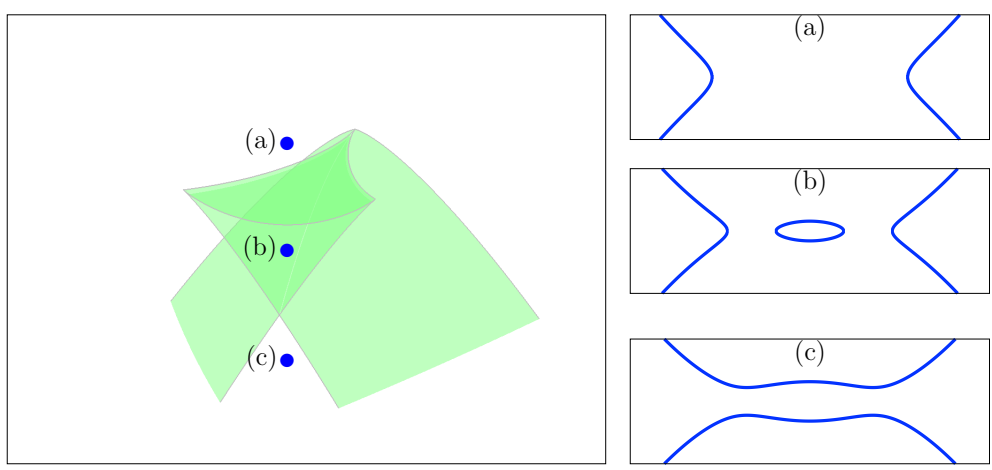

Fig. 9 A replica of the bifurcation surface of a bifurcation problem case from [24] (case $8^{-}$, page 208) showing the possibility for a bubble to emerge (panel (b)); compare with figures 3(c) and 4(c). The associated bifurcation equation with distinguished parameters is: $x^{2}-\lambda^{4}+\alpha+\beta \lambda+\gamma \lambda^{2}=0$ where $\lambda$ is the distinguished parameter and $(\alpha, \beta, \gamma)$ are three unfolding parameters. Taking different values for the triple of unfolding parameters give the three dots labelled (a), (b) and (c) on the left panel, which give topologically different transversal intersections separated by bifurcations.

\section{A Singularity theory approach}

We build upon our previous studies that established that inflection sets and their topological shape changes (parameterised by a distinguished parameter expressed in the context of bifurcation singularity theory by Golubitsky and Schaeffer) can characterise and classify specific classes of canard mediated transitions. We extend our studies and show that a wider class of canards, namely canards mediated by folded-singularities can also be studied by the same framework. To unveil more information about the relationship between inflection sets and the dynamics near a folded singularity (saddle or node), it is useful to evaluate the inflection equation close to the folded singularity and along time instants $t$. To this end, we conveniently write equation (18) by completing the square yielding

$$
\bar{h}^{2}-\frac{1}{4}\left[\varepsilon \mu-2 x\left(\mu t+z_{0}+x\right)\right]^{2}+\varepsilon\left(\mu t+z_{0}+x\right)^{2}=0,
$$

with

$$
\bar{h}=h+\frac{1}{2}\left(\varepsilon \mu-2 x\left(\mu t+z_{0}+x\right)\right),
$$

which is equivalently rewritten as follows

$$
\bar{h}^{2}-\left(\sqrt{\frac{\varepsilon \mu}{2}}\right)^{4}+\left(\mu t+z_{0}+x\right)^{2}\left(\varepsilon-x^{2}\right)+\left(\sqrt{\frac{\varepsilon \mu}{2}}\right)^{2} 2 x\left(\mu t+z_{0}+x\right)=0 .
$$

Following from our previous studies, we recast equation (38) as a bifurcation problem with a distinguished parameter, which leads to the case $8^{-}$(of the singularity theory) on page 208 of [24] as follows

$$
X^{2}-\lambda^{4}+\alpha+\beta \lambda+\gamma \lambda^{2},
$$


with

$$
\begin{aligned}
X & =\bar{h} \\
\lambda & =\sqrt{\frac{\varepsilon \mu}{2}} \\
\alpha & =\left(\mu t+z_{0}+x\right)^{2}\left(\varepsilon-x^{2}\right), \\
\beta & =0 \\
\gamma & =2 x\left(\mu t+z_{0}+x\right) .
\end{aligned}
$$

The topological shape of the solution to equation (39) is shown on figure 9. The figure also shows the topological changes as a bifurcation parameter is varied. In particular, it shows that when $\mu>0$ (i.e. in the folded node case) an additional closed component of the inflection curve emerges, which corresponds to the inflection bubble that we have studied in the present work and it is in contrast to the planar case where only a single point of this bubble exists, namely the equilibrium point.

Acknowledgements SR would like to acknowledge Ikerbasque (The Basque Foundation for Science). Moreover, SR and JUA would like to thank for the fact that this research is supported by the Basque Government through the BERC 2018-2021 program and by the Spanish State Research Agency through BCAM Severo Ochoa excellence accreditation SEV2017-0718 and through project RTI2018-093860-B-C21 funded by (AEI/FEDER, UE) and acronym "MathNEURO". MD acknowledges BCAM's hospitality during a visiting fellowship in the summer 2019.

\section{References}

1. R. Amir, M. Michaelis and M. Devor, Burst discharge in primary sensory neurons: triggered by subthreshold oscillations, maintained by depolarizing afterpotentials, J. Neurosci. 22(3), 1187-1198, 2002.

2. V. I. Arnol'd, Contact geometry: the geometrical method of Gibbs's thermodynamics, In: D. G. Caldi and G. D. Mostow, Eds., Proceedings of the Gibbs Symposium (Yale University, New Haven, CT, May 15-17, 1989), Amer. Math. Soc. and Amer. Inst. Physics, pp. 163-179, 1990.

3. V. I. Arnol'd, V. S. Afrajmovich, Y. S. $\mathrm{Il}^{\prime}$ yashenko and L. P. Shil'nikov, Dynamical systems $V$ : bifurcation theory and catastrophe theory, Encyclopaedia of Mathematical Sciences vol. 5, Springer-Verlag, Berlin, 1994.

4. S. M. Baer, T. Erneux and J. Rinzel, The slow passage through a Hopf bifurcation: delay, memory effects, and resonance, SIAM J. Appl. Math. 49(1): 55-71, 1989.

5. D. Barkley, Slow manifolds and mixed-mode oscillations in the Belousov-Zhabotinskii reaction, J. Chem. Phys. 89(9): 5547-5559, 1988.

6. É. Benoît, J.-L. Callot, F. Diener and M. Diener, Chasse au canard, Coll. Math. 32(1-2): 37-119, 1981.

7. É. Benoît, Canards et enlacements, Publ. Math. IHÉS 72(1): 63-91, 1990.

8. É. Benoît and Lobry, C., Les canards de $\mathbb{R}^{3}$, CR Acad. Sci. Paris 294, 483-488, 1982.

9. É Benoît, M. Brøns, M. Desroches and M. Krupa, Extending the zero-derivative principle for slow-fast dynamical systems, Z Angew Math Phys 66(5): 2255-2270, 2015.

10. N. Berglund, Adiabatic dynamical systems and hysteresis, PhD thesis, EPFL (Lausanne, Switzerland), 1998, url: https://infoscience.epfl.ch/record/32277.

11. M. Brøns, Bifurcations and instabilities in the Greitzer model for compressor system surge, Math. Eng. Ind. 2(1): 51-63, 1988.

12. M. Brøns and K. Bar-Eli, Asymptotic analysis of canards in the EOE equations and the role of the inflection line, Proc. Roy. Soc. A, 445(1924): 305-322, 1994.

13. M. Brøns, M. Krupa and M. Wechselberger, Mixed mode oscillations due to the generalized canard phenomenon, Fields Inst. Commun. 49: 39-63, 2006. 
14. M. Desroches, B. Krauskopf and H. M. Osinga, Mixed-mode oscillations and slow manifolds in the self-coupled FitzHugh-Nagumo system, Chaos 18(1): 015107, 2008.

15. M. Desroches and M. R. Jeffrey, Canards and curvature: the 'smallness of $\varepsilon$ ' in slow-fast dynamics, Proc. Roy. Soc. A 467(2132): 2404-2421, 2011.

16. M. Desroches, M. Krupa and S. Rodrigues, Spike-adding in parabolic bursters: the role of folded-saddle canards, Physica D 331: 58-70, 2016.

17. M. Desroches, M. Krupa and S. Rodrigues, Inflection, canards and excitability threshold in neuronal models, J. Math. Biol. 67(4): 989-1017, 2013.

18. M. Desroches, J. Guckenheimer, B. Krauskopf, C. Kuehn, H. M. Osinga and M. Wechselberger, Mixed-mode oscillations with multiple time scales, SIAM Review 54(2) (2012) $211-288$.

19. M. Desroches, A. Guillamon, E. Ponce, R. Prohens, S. Rodrigues and A. E. Teruel, $C a-$ nards, folded nodes, and mixed-mode oscillations in piecewise-linear slow-fast systems, SIAM Rev. 58(4): 653-691, 2016.

20. F. Dumortier and R. Roussarie, Canard cycles and center manifolds, Mem. Amer. Math. Sco., vol. 577, Amer. Math. Soc., Providence, 1996.

21. W. Eckhaus, Relaxation oscillations including a standard chase on French ducks, In Asymptotic Analysis II, F. Verhulst, Ed., Lect. Notes in Math. vol. 985, SpringerVerlag, Berlin, 1983, pp. 449-497.

22. N. Fenichel, Geometric singular perturbation theory for ordinary differential equations, J. Diff. Eq. 31(1): 53-98, 1979.

23. J.-M. Ginoux, B. Rossetto and L. O. Chua, Slow invariant manifolds as curvature of the flow of dynamical systems, Int. J. Bif. Chaos 18(11): 3409-3430, 2008.

24. M. Golubitsky and D. G. Schaeffer, Singularities and Groups in Bifurcation Theory, Applied Mathematical Sciences vol. 51, Springer-Verlag, Berlin, 1985.

25. M. Golubitsky, K. Josić and T. J. Kaper, An unfolding theory approach to bursting in fast-slow systems, In: Global analysis of dynamical systems, H. W. Broer, B. Krauskopf and G. Vegter, Eds., IoP Publishing Ltd, Bristol, 2001, pp. 277-308.

26. A. N. Gorban and I. V. Karlin, Method of invariant manifold for chemical kinetics, Chemical Engineering Science 58(21): 4751-4768, 2003.

27. M. G. Hayes, T. J. Kaper, P. Szmolyan and M. Wechselberger, Geometric desingularization of degenerate singularities in the presence of fast rotation: A new proof of known results for slow passage through Hopf bifurcations, Ind. Math. 27(5): 1184-1203, 2016.

28. P. Heiter and D. Lebiedz, Towards differential geometric characterization of slow invariant manifolds in extended phase space: Sectional curvature and flow invariance, SIAM J. Appl. Dyn. Syst. 17(1): 732-753, 2018.

29. C. K. R. T. Jones, Geometric singular perturbation theory, In: Dynamical systems, C.I.M.E. Lectures, Montecatini Terme (Italy), R. Johnson, Ed., Lect. Notes in Math. vol. 1609, Springer, Berlin, 1995, pp. 44-118.

30. T. Koos, J. M. Tepper, C. J. Wilson, Comparison of IPSCs evoked by spiny and fastspiking neurons in the neostriatum, J. Neurosci. 24(36): 7916-22, 2004.

31. M. Krupa and P. Szmolyan, Relaxation oscillation and canard explosion, J. Diff. Eq. 174(2): 312-368, 2001

32. M. Krupa and M. Wechselberger, Local analysis near a folded saddle-node singularity, J. Diff. Eq. 248(12): 2841-2888, 2010.

33. F. Marino, M. Ciszak, S. F. Abdalah, K. Al-Naimee, R. Meucci and F. T. Arecchi, Mixed-mode oscillations via canard explosions in light-emitting diodes with optoelectronic feedback, Phys. Rev. E 84(4): 047201, 2011.

34. W. Marszalek, Fold points and singularity induced bifurcation in inviscid transonic flow, Phys. Lett. A 376(28-29): 2032-2037, 2012.

35. J. Masełko and H. L. Swinney, Complex periodic oscillations and Farey arithmetic in the Belousov-Zhabotinskii reaction, J. Chem. Phys. 85(11): 6430-6441, 1986.

36. J. Mitry and M. Wechselberger, Folded saddles and faux canards, SIAM J. Appl. Dyn. Syst. 16(1): 546-596, 2017.

37. A. I. Neishtadt, Asymptotic investigation of the loss of stability as a pair of eigenvalues slowly cross the imaginary axis, Uspekhi Mat. Nauk. 40: 190-191, 1985.

38. A. J. Nevado-Holgado, F. Marten, M. P. Richardson, J. R. Terry, Characterising the dynamics of EEG waveforms as the path through parameter space of a neural mass model: Application to epilepsy seizure evolution NeuroImage 59 (3), 2374, 2012. 
39. M. Okuda, Inflector analysis of the second stage of the transient phase for an enzymatic one-substrate reaction, Progr. Theor. Phys. 68(6): 1827-1840, 1982.

40. B. Peng, V. Gáspár and K. Showalter, False bifurcations in chemical systems: canards, Phil. Trans. Roy. Soc. A 337(1646): 275-289, 1991.

41. S. Peron and F. Gabbiani, Spike frequency adaptation mediates looming stimulus selectivity in a collision-detecting neuron, Nat. Neurosci. 12(3): 318-326, 2009.

42. S. Rajesh and G. Ananthakrishna, Relaxation oscillations and negative strain rate sensitivity in the Portevin? Le Chatelier effect, Phys. Rev. E 61(4): 3664, 2000.

43. J. Rubin and M. Wechselberger, The selection of mixed-mode oscillations in a HodgkinHuxley model with multiple timescales, Chaos 18(1): 015105, 2008.

44. S. Rodrigues, D. Barton, R. Szalai, O. Benjamin, M. P. Richardson and J. R. Terry, Transitions to spike-wave oscillations and epileptic dynamics in a human corticothalamic mean-field model, J. of Computational Neuroscience 27(3): 507-526, 2009.

45. S. Rodrigues, D. Barton, F. Marten, M. Kibuuka, G. Alarcon, M. P. Richardson and J. R. Terry, A method for detecting false bifurcations in dynamical systems: Application to neural-field models, Biological Cybernetics, 102(2): 145-154, 2010.

46. F. Marten, S. Rodrigues, O. Benjamin, M. P. Richardson and J. R. Terry, Onset of polyspike complexes in a mean-field model of human EEG and its application to absence epilepsy, Philosophical Transactions of the Royal Society A, 367(1891): 1145-1161, 2009.

47. R. E. Plant, Bifurcation and resonance in a model for bursting nerve cells, Journal of Mathematical Biology 11(1): 15-32, 1981

48. M. A. Shishkova, A discussion of a certain system of differential equations with a small parameter multiplying the highest derivatives, Dokl. Akad. Nauk SSSR 209: 576-?579, 1973.

49. P. Szmolyan and M. Wechselberger, Canards in $\mathbb{R}^{3}$, J. Diff. Eq. 177(2): 419-453, 2001.

50. M. Wechselberger, Existence and bifurcation of canards in $\mathbb{R}^{3}$ in the case of a folded node, SIAM J. Appl. Dyn. Syst. 4(1): 101-139, 2005.

51. M. Wechselberger, A propos de canards (apropos canards), Trans. Amer. Math. Soc. 364(6): 3289-3309, 2012.

52. E. C. Zeeman, Levels of structure in catastrophe theory illustrated by applications in the social and biological sciences, In: Proc. Int. Congr. Math., Vancouver (Canada), 1974, vol. 2, pp. 533-548. 